\title{
Alterações orais em três espécies de Leontopithecus mantidas em cativeiro (Callitrichidae, Primates)
}

\section{Dental changes in three species of Leontopithecus maintained in captivity (Callitrichidae, Primates)}

\author{
Carlos Henrique de F. Burity ${ }^{1,2}$, Maria Urânia Alves ${ }^{3}$, Alcides Pissinatti ${ }^{4}$
}

\section{Resumo}

Foram estudadas as alterações orais que ocorrem em primatas não humanos, cativos, do gênero Leontopithecus, suas diferenças entre espécies, suas freqüências e a influência do cativeiro. Sessenta crânios de Leontopithecus, do acervo do Museu Primatológico do Centro de Primatologia do Rio de Janeiro (CPRJ-FEEMA), foram examinados. O teste do $\chi^{2}$ foi utilizado para avaliar as diferenças nas incidências das alterações orais e o coeficiente de correlação de Pearson para o estudo da correlação entre o número de alterações e o tempo de cativeiro dos animais. Entre as três espécies estudadas, L. chrysomelas, $L$. rosaliae $L$. chrysopygus, as diferenças nas alterações orais foram estatisticamente significativas para cáries, crazing, doença pulpar e maloclusão. O complexo caninoincisivo foi mais afetado do que a série molar. A incidência de alterações orais aumentou à medida que aumentou o tempo de cativeiro dos micos e diferiu nas três espécies de Leontopithecus estudadas, sendo L. chrysopygusi a mais suscetível.

Palavras chave: Leontopithecus; mico-leão; alterações orais; cativeiro

\section{Introdução}

Leontopithecus (Lesson, 1840) pertence à família Callitrichidae, subordem Anthropoidea e infra-ordem Platyrrhini. Na família compartilha características tais como menor tamanho corporal; unhas semelhantes a garras nos dedos, exceto no hallux; perda do terceiro molar na mandíbula e na maxila (32 dentes) e freqüência alta de gêmeos dizigóticos (Martin, 1990).

O gênero é caracterizado na família como o que possui formas mais robustas e pelo pelame longo e sedoso com tons de dourado-avermelhado a dourado-amarelado (variando em grau nas três formas) e áreas escuras do pardo escuro ao negro retinto. A face, quase nua, é rodeada por um tipo de juba. A mão e os dedos são longos e possuem uma membrana unindo parcialmente os dedos medianos da mão (Coimbra-Filho, 1976).
O uso de calitriquídeos em pesquisas biomédicas foi amplamente difundido pela sua suscetibilidade a diversas doenças e pela facilidade da sua manutenção em cativeiro (espaço, dieta e manejo) (Cicmanec, 1978; Box, 1995).

Se primatas não-humanos, ao invés de roedores, tivessem sido usados para testar a teratogenicidade da talidomida durante o seu desenvolvimento nas décadas de 40 e 50 , as causas adversas em fetos humanos teriam sido detectadas (King, 1995).

Uma tendência atual é tornar o cativeiro o mais saudável possível, e para isso é necessário deter informações suficientes sobre comportamento, fisiologia, imunologia e patologia nas diversas condições que apresenta (Box, 1995; Markowitz e Gavazzi, 1995).

O tema de patologias orais tem recebido na literatura um número menor de publicações do que o devido (Levy, 1976; Hershkovitz, 1977; Smith et al., 1977) uma vez que os animais cativos são mais suscetíveis a patologias do que os animais silvestres (Devoto et al., 1970; Crovella e Ardito, 1994).

Espécimes silvestres de L. rosalia apresentando alterações orais, atribuídas, provavelmente, a problemas de consangüinidade já foram reportados na literatura (Pinder e Pissinatti, 1991).

A alta prevalência de alterações orais em primatas cativos pode resultar em via de acesso a infecçōes secundárias, dificuldades na alimentação e dificuldades na manutenção em cativeiro.

O objetivo deste estudo foi avaliar as alterações orais em três espécies de Leontopithecus cativos, as possiveis diferenças interespecíficas e a influência do tempo de cativeiro.

\section{Material e Métodos}

Foram utilizados 60 crânios de micos-leões (Leontopithecus): 15 L. chrysomelas, 20 L. rosalia e $25 \mathrm{~L}$. chrysopygus, do acervo do Museu Primatológico do Centro de Primatologia do Rio de Janeiro (CPRJ-FEEMA) (Coimbra-Filho et al., 1986). Todos os animais eram adultos, de ambos os sexos, e foram mantidos sob as mes-

\footnotetext{
${ }^{1}$ Departamento de Anatomia, IB, Universidade do Estado do Rio de Janeiro (UERJ)

2 Pós-Graduação em Biociências Nucleares (Biologia), IB, UERJ

${ }^{3}$ Odontopediatria, Universidade de Nova Iguaçu

${ }^{4}$ Centro de Primatologia do Rio de Janeiro (CPRJ-FEEMA), R. Fonseca Teles 121 s/1624, 20940-200 Rio de Janeiro, RJ, Brasil
} 
mas condições de cativeiro no CPRJ-FEEMA (CoimbraFilho et al., 1981). Os crânios foram examinados sob lupa esteroscópica (10x) para identificação das alterações orais.

$O$ teste do $\chi^{2}$ foi utilizado para avaliar as diferenças entre as incidências das alterações orais nas três espécies e o coeficiente de correlação de Pearson para avaliar a relação do número de alterações orais com o tempo de cativeiro.

\section{Resultados}

As alterações orais observadas foram classificadas de acordo com a descrição de Smith et al. (1977): cáries, maloclusão, tártaro, doença periodontal, doença pulpar, crazing e atrito excessivo. Uma alteração oral ainda não descrita na literatura foi reportada: a alteração de cor, caracterizada pelo esmalte brilhante com aspecto nacarado.

A Tabela mostra a incidência de cada alteração oral nas três espécies de Leontopithecus. Algumas alterações apresentaram ocorrências estatisticamente diferentes entre as três espécies: cáries, crazing, doença pulpar e maloclusão. As diferenças observadas nas outras alterações não foram estatisticamente significantes. A doença periodontal foi a mais freqüentemente observada.

A Fig. 1 mostra a incidência de crazing nos dentes da maxila e da mandíbula nas três espécies. Na mesma figura pode ser observada a maior incidência de alterações no complexo canino-incisivo em relação às alterações na série molar.

A Fig. 2 mostra o resultado da correlação entre o número de alterações e o tempo de cativeiro para as três espécies com uma correlação média $(r=0,57)$ significativa: $p<0,0001$, indicando assim um aumento do número de alterações orais com o passar do tempo de cativeiro.

\section{Discussão}

Alterações orais foram observadas nos micos (Leontopithecus) mantidos em cativeiro.

Em algumas, como a cárie, a etiologia tem sido atribuída a três fatores: a) microflora da cavidade oral; b) dieta; c)
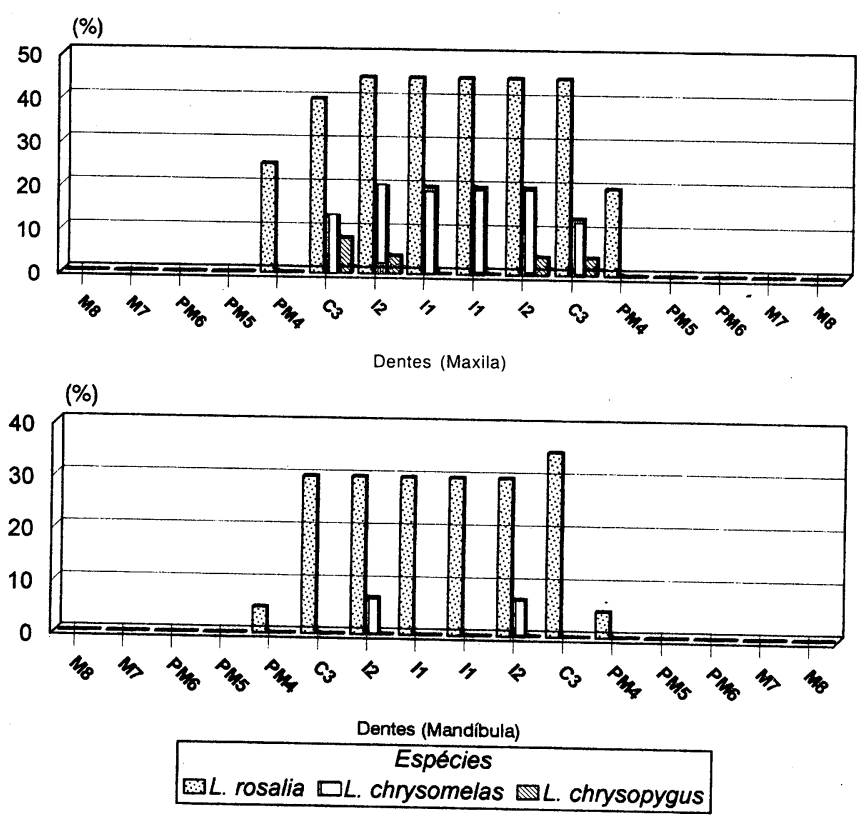

Figura 1 - Gráfico de barras ilustrando a freqüência de crazing nos dentes da maxila e da mandíbula, comparando as três espécies de Leontopithecus.

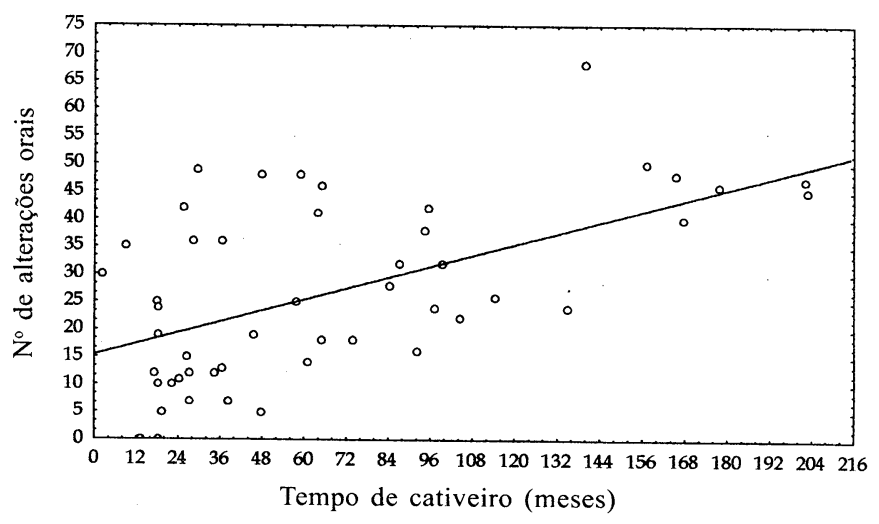

Figura 2 - Correlação entre o número de alterações orais e o tempo de cativeiro para as três espécies de Leontopithecus (unidas).

Tabela - Incidência das alterações orais observadas nas três espécies de Leontopithecus, e o nível de significância das diferenças entre as espécies

\begin{tabular}{|c|c|c|c|c|}
\hline Alterações orais & L. chrysomelas $(n=15)$ & L. rosalia $(n=20)$ & L. chrysopygus $(\mathrm{n}=25)$ & Total $(n=60)$ \\
\hline Cárie & $6 / 15=40 \%$ & $12 / 20=60 \%$ & $22 / 25=80 \%{ }^{* *}$ & $40 / 60=67 \%$ \\
\hline Maloclusão & $5 / 15=23 \%$ & $4 / 20=20 \%$ & $14 / 25=56 \%$ & $23 / 60=38 \%$ \\
\hline Tártaro & $10 / 15=67 \%$ & $16 / 20=80 \%$ & $23 / 25=92 \%$ & $49 / 60=82 \%$ \\
\hline Crazing & $3 / 15=20 \%$ & $10 / 20=50 \%$ & $2 / 25=8 \%$ & $15 / 60=25 \%$ \\
\hline Doença periodontal & $13 / 15=87 \%$ & $18 / 20=90 \%$ & $21 / 25=84 \%$ & $52 / 60=87 \%$ \\
\hline Doença pulpar & $2 / 15=13 \%$ & $4 / 20=20 \%$ & $15 / 25=60 \%{ }^{* *}$ & $21 / 60=35 \%$ \\
\hline Atrito excessivo & $6 / 15=40 \%$ & $6 / 20=30$ & $5 / 25=20 \%$ & $17 / 60=28 \%$ \\
\hline Alteração de cor & $5 / 15=33 \%$ & $5 / 20=25 \%$ & $3 / 25=12 \%$ & $13 / 60=22 \%$ \\
\hline
\end{tabular}

* $p<0.05 ;{ }^{* \star}-p<0.01$ 
quantidade e pH da secreção salivar (Smith et al., 1977).

As condições de cativeiro e dieta foram mantidas constantes durante a permanência dos espécimes estudados em cativeiro (Coimbra-Filho e Maia, 1977; Coimbra-Filho et al., 1981).

A maloclusão pode ter sido resultado de alterações orais anteriores como perda de dimensão vertical e mordida cruzada, entre outras. A alta incidência de maloclusão tem sido associada também com a perda das estruturas dentais por lesões cariosas (McDonald, 1974).

Ressaltando que a dieta foi a mesma para os símios, e que os mesmos possuem aparatos mandibulares semeIhantes (Burity, 1995), a causa provável para as diferenças estatisticamente significativas na incidência do crazing entre as espécies ainda não foi determinada.

Hershkovitz (1977) atribuiu a presença do crazing ao stress de mastigação entre as duas arcadas, o que seria pouco provável se considerarmos somente este stress como causador, ao invés de um conjunto de fatores ainda não determinados mais o stress de mastigação.

A incidência de alterações orais em Leontopithecus é maior no complexo canino-incisivo do que na série molar (Fig. 1). No entanto, Devoto et al. (1970) reportaram uma maior freqüência no complexo molar ao invés de no complexo canino-incisivo em outros primatas não humanos.

A doença periodontal foi a alteração oral mais freqüente nos animais estudados, contudo, ela aparece em segundo lugar, após o tártaro, em estudos anteriores com outros primatas (Crovella e Ardito, 1994).

Diniz e Costa (1995) reportam os problemas de saúde mais freqüentes encontrados em uma colônia de Callithrix jacchus. Dentre eles os digestivos se mostraram entre os primeiros seguidos dos dentais. Este relato é importante, uma vez que com o aumento do tempo de cativeiro aumenta a possibilidade do animal desenvolver alterações orais.

Toda instituição que mantém primatas cativos deveria dar maior atenção a estas alterações, não só pela perda de apetite e apatia causada nos animais, mas também por ser uma via de acesso a infecções secundárias.

Em conclusão, para se manter uma colônia de micos saudáveis em cativeiro é necessário conhecê-los um pouco mais, até mesmo sua cavidade oral, uma vez que animais nas mesmas condições de cativeiro e dieta podem apresentar freqüências distintas de alterações orais. Isto nos leva a pensar nas hipóteses para explicar estas diferenças: será uma diferença na microflora? ou na secreção salivar?

\section{Agradecimentos}

Ao Grupo Coffin (Refrigerantes Niterói SA) pela constante ajuda ao projeto Centro de Primatologia do Rio de Janeiro. Ao Dr. Benjamin Beck (National Zoological Park, Washington D.C.) e ao Dr. Andrew Baker (The Zoological Society of Philadelphia) pela sempre providencial ajuda com a literatura.

\section{Abstract \\ Dental changes in three species of Leontopithecus maintained in captivity (Callitrichidae, Primates)}

The dental changes occurring in captive non-human primates of the genus Leontopithecus and its differences among three species, $L$. chrysomelas, $L$. rosalia and $L$. chrysopygus, regarding frequency and captivity influence, were studied. Sixty skulls of the Leontopithecus, from the collection of the Museum of Centro de Primatologia do Rio de Janeiro (CPRJ-FEEMA) were examined. The $\chi^{2}$ test was used to evaluate the differences between incidence of changes observed and Pearson's coefficient of correlation to evaluate the number of changes observed and the time of captivity. The differences among the three species were significant for caries, crazing, pulpar disease and malocclusion. The complex canine-incisors were more affected than the molars. The incidence of oral changes was high in animals maintained more time in captivity conditions and was different in the three species of the Leontopithecus studied; L. chrysopygus was the most susceptible.

Key words: Leontopithecus; tamarins; dental changes; captivity

\section{Referências bibliográficas}

Box HO 1995. Biological propensities of the Callitrichidae: a much used-little known group. Lab Animals 29: 237-243.

Burity CHF 1995. Craniometria em três espécies de Leontopithecus Lesson, 1840 (Callitrichidae,Primates): análise multivariada. Tese de Mestrado. Universidade do Estado do Rio de Janeiro, Rio de Janeiro, 118 pp.

Cicmanec J 1978. Medical problems encountered in a Callitrichidae colony, p. 331-336. In DG Kleiman, The Biology and Conservation of the Callitrichidae. Smithsonian Institution Press, Washington, DC.

Coimbra-Filho AF 1976. Os saguis do gênero Leontopithecus Lesson, 1840 - (Callithricidae - Primates). Tese de Mestrado. Universidade Federal do Rio de Janeiro, Rio de Janeiro, 72 pp.

Coimbra-Filho AF, Maia AA 1977. Alimentação de saguis em cativeiro. IBDF. Brasil Florestal 29: 15-26.

Coimbra-Filho AF, Silva RR, Pissinatti A 1981. Sobre a dieta de Callitrichidae em cativeiro. Rev Biotérios 1: 83-93.

Coimbra-Filho AF, Pissinatti A, Silva RR 1986. $\mathcal{O}$ acervo do Museu de Primatologia (CPRJ-FEEMA), p. 505-514. In Milton Thiago de Mello, A Primatologia no Brasil, 2a. ed., SBPr, Campinas.

Crovella S, Ardito G 1994. Frequencies of oral pathologies in a sample of 767 non-human primates. Primates 35: 225230.

Devoto FCH, Moreno Rios RN, Schejtman R 1970. Lesiones de las apofisis alveolares en monos sudamericanos. Acta Zoologica Lilloana XXVII: 109-121.

Diniz LSM, Costa EO 1995. Health problems of Callithrix jacchus in captivity. Braz J Med Biol Res 28: 61-64. 
Hershkovitz P 1977. Living New World Monkeys, Vol. 1. University Chicago Press, Chicago, $1117 \mathrm{pp}$.

King FA 1995. Nonhuman Primates in research: A review of their crucial role. Lab Animal January: 28-32.

Levy BM 1976. Chronic destructive periodontitis in marmosets. Am J Pathol 83: 637-640.

Markowitz H, Gavazzi A 1995. Eleven principles for improving the quality of captive animal life. Lab Animal April: 30-33.

Martin RD 1990. Primate Origins and Evolution a Phylogenetic Reconstruction. Chapman and Hall, London, $804 \mathrm{pp}$.

McDonald RE 1974. Dentitry for the child and adolescent, 2nd ed., CV Mosby Company, Saint Louis, 350 pp.

Newbrun E 1983. Cariology. Williams and Wilkins, Baltimore, $255 \mathrm{pp}$.

Pinder L, Pissinatti A 1991. Malformações congênitas em Leontopithecus rosalia (Linnaeus, 1766) (Callitrichidae, Primates), p. 191-196. In A Rylands, AT Bernardes (eds.), A Primatologia no Brasil. 3., Fundação Biodiversitas, Belo Horizonte.

Smith JD, Genoways HH, Knox Jones Jr J 1977. Cranial and dental anomalies in three species of Platyrrhine monkeys from Nicarágua. Folia Primatol 28: 1-42. 